\title{
DISCONTINUITIES IN AN AXISYMMETRIC GENERALIZED THERMOELASTIC PROBLEM
}

\author{
MONCEF AOUADI
}

Received 11 August 2004 and in revised form 14 February 2005

This paper deals with discontinuities analysis in the temperature, displacement, and stress fields of a thick plate whose lower and upper surfaces are traction-free and subjected to a given axisymmetric temperature distribution. The analysis is carried out under three thermoelastic theories. Potential functions together with Laplace and Hankel transform techniques are used to derive the solution in the transformed domain. Exact expressions for the magnitude of discontinuities are computed by using an exact method developed by Boley (1962). It is found that there exist two coupled waves, one of which is elastic and the other is thermal, both propagating with finite speeds with exponential attenuation, and a third which is called shear wave, propagating with constant speed but with no exponential attenuation. The Hankel transforms are inverted analytically. The inversion of the Laplace transforms is carried out using the inversion formula of the transform together with Fourier expansion techniques. Numerical results are presented graphically along with a comparison of the three theories of thermoelasticity.

\section{Introduction}

Much attention has been devoted to the generalization of the equations of coupled thermoelasticity due to Biot [1]. This is mainly due to the fact that the heat equation of this theory is parabolic, and hence automatically predicts infinite speed of propagation for heat waves. Clearly, this contradicts physical observations that the maximum wave speed cannot exceed that of light in vacuum. During the last three decades, nonclassical theories have been developed to remove this paradox. Lord and Shulman [13] introduced the theory of generalized thermoelasticity with one relaxation time. This theory is based on a new law of heat conduction to replace Fourier's law. The heat equation is replaced by a hyperbolic one which ensures finite speeds of propagation for heat and elastic waves. Green and Lindsay [8] have developed a temperature-rate-dependent thermoelasticity by including temperature rate among the constitutive variables, which does not violate the classical Fourier laws of heat conduction when the body under consideration has a center of symmetry. This theory also predicts a finite speed of heat propagation. Both generalized theories consider heat propagation as a wave phenomenon rather than a diffusion 
phenomenon. In view of the exponential evidence available in favor of finiteness of heat propagation speed, generalized thermoelasticity theories are supposed to be more realistic than the conventional theory in dealing with practical problems involving very large heat fluxes and/or short time intervals, like those occurring in laser units and energy channels.

Furukawa et al. $[6,7]$ applied the theory of thermoelasticity to an infinite cylindrical body. Jordan and Puri in [12] used Boley's method [2] to find the magnitude of discontinuities. Ignaczak in $[10,11]$ studied a strong discontinuity wave and obtained a decomposition theorem. Chandrasekharaiah and Srinath in [4] studied the propagation of discontinuities within the context of thermoelasticity without energy dissipation. These authors employed the Laplace transform to obtain small-time approximations for the field functions. For earlier related research on axisymmetric thermoelastic problems using the Lord-Shulman model, see the papers of Rossikhin et al. [18] and Orisamolu et al. [16].

The aim of this paper is to investigate the propagation of discontinuities not only in the stress but also in the temperature and displacement fields. Exact expressions for the magnitude in these quantities are also given. In addition, numerical methods are used to invert the integral transforms and to evaluate the improper integrals involved to obtain the solution in the physical domain. Based on the analysis of discontinuities and numerical results, a comparison of the three theories of thermoelasticity is given. The results given here are also compared with those of other investigators.

\section{Formulation of the problem}

We consider a homogeneous isotropic thermoelastic thick plate of height $2 h$ and of infinite extent. The conditions of the problem are assumed to be axisymmetric. We will take the axis of symmetry to be the $z$-axis and the origin of the system of coordinates at the midpoint between the lower and upper surfaces of the plate. By denoting the cylindrical polar coordinates as $(r, \phi, z)$, we study the problem in the region $\Omega$ defined by

$$
\Omega=\{(r, \varphi, z): 0 \leq r \leq \infty, 0 \leq \phi \leq 2 \pi,-h<z<h\} .
$$

The stress-strain relations have the form

$$
\begin{aligned}
\sigma_{r r} & =2 \mu \frac{\partial u}{\partial r}+\lambda e-\gamma\left(T-T_{0}+\nu \frac{\partial T}{\partial t}\right), \\
\sigma_{\phi \phi} & =2 \mu \frac{u}{r}+\lambda e-\gamma\left(T-T_{0}+\nu \frac{\partial T}{\partial t}\right), \\
\sigma_{z z} & =2 \mu \frac{\partial w}{\partial z}+\lambda e-\gamma\left(T-T_{0}+\nu \frac{\partial T}{\partial t}\right), \\
\sigma_{r z} & =\mu\left(\frac{\partial u}{\partial z}+\frac{\partial w}{\partial r}\right), \quad \sigma_{r \phi}=\sigma_{z \phi}=0,
\end{aligned}
$$

where $\lambda, \mu$ are Lamé's constants, $\nu$ is a relaxation time, $\gamma$ is a material constant given by $\gamma=(3 \lambda+2 \mu) \alpha_{t}, \alpha_{t}$ is the coefficient of linear thermal expansion, $T_{0}$ is a reference 
temperature chosen such that $\left|\left(T-T_{0}\right) / T_{0}\right| \ll 1$, and $e$ is the dilatation given by

$$
e=\frac{\partial u}{\partial r}+\frac{u}{r}+\frac{\partial w}{\partial z}
$$

The equations of motion can be written as

$$
\begin{gathered}
\rho \frac{\partial^{2} u}{\partial t^{2}}=\mu \nabla^{2} u-\frac{\mu}{r^{2}} u+(\lambda+\mu) \frac{\partial e}{\partial r}-\gamma\left(1+\nu \frac{\partial}{\partial t}\right) \frac{\partial T}{\partial r} \\
\rho \frac{\partial^{2} w}{\partial t^{2}}=\mu \nabla^{2} w+(\lambda+\mu) \frac{\partial e}{\partial z}-\gamma\left(1+\nu \frac{\partial}{\partial t}\right) \frac{\partial T}{\partial z}
\end{gathered}
$$

where $\rho$ is the density, and the Laplacian operator $\nabla^{2}$ is given by

$$
\nabla^{2}=\frac{\partial^{2}}{\partial r^{2}}+\frac{1}{r} \frac{\partial}{\partial r}+\frac{\partial^{2}}{\partial z^{2}} .
$$

The generalized equation of heat conduction has the form

$$
k \nabla^{2} T=\rho C_{E}\left(\frac{\partial}{\partial t}+\tau_{0} \frac{\partial^{2}}{\partial t^{2}}\right) T+T_{0} \gamma\left(\frac{\partial}{\partial t}+n_{0} \tau_{0} \frac{\partial^{2}}{\partial t^{2}}\right) e
$$

where $k$ is the coefficient of thermal conductivity, $C_{E}$ is the specific heat at constant strain, and $\tau_{0}$ is another relaxation time. The use of the symbol $n_{0}$ in (2.6) makes these fundamental equations possible for three different theories of thermoelasticity. For the Lord-Shulman (LS) theory, $\tau_{0}>0, \nu=0, n_{0}=1$; for the Green-Lindsay (GL) theory, $\nu \geq \tau_{0}>0, n_{0}=0$; and for the classical (CT) theory, $n_{0} \tau_{0}=\tau_{0}=\nu=0$. There exist the following differences between the two generalized theories.

(i) The LS theory involves one relaxation time of thermoelastic process $\left(\tau_{0}\right)$, and that of GL theory involves two relaxation times $\left(\tau_{0}, \nu\right)$.

(ii) The LS energy equation involves first and second time derivatives of strain, whereas the corresponding equation in GL theory needs only the first time derivative of strain.

(iii) In the linearized case, according to the approach of Green and Lindsay, heat cannot propagate with finite speed unless the stresses depend on the temperature rate, whereas according to Lord and Shulman, the heat can propagate with finite speed even though the stresses there are independent of the temperature velocity.

Now we introduce the nondimensional variables

$$
\begin{gathered}
r^{*}=\eta_{0} r, \quad z^{*}=\eta_{0} z, \quad u^{*}=\eta_{0} u, \quad w^{*}=\eta_{0} w, \\
\theta=\frac{\gamma\left(T-T_{0}\right)}{\lambda+2 \mu}, \quad \sigma_{i j}^{*}=\frac{\sigma_{i j}}{\mu}, \quad t^{*}=c_{0} \eta_{0} t, \quad \tau_{0}^{*}=c_{0} \eta_{0} \tau_{0}, \quad \nu^{*}=c_{0} \eta_{0} \nu,
\end{gathered}
$$

where $\eta_{0}=\rho c_{0} C_{E} / k$ is the dimensionless characteristic length and $c_{0}=\sqrt{(\lambda+2 \mu) / \rho}$ is the velocity of longitudinal wave. In terms of these nondimensional variables, (2.2), (2.3), 
1018 Discontinuities in an axisymmetric thermoelastic problem

and (2.6) take the form (dropping the asterisks for convenience)

$$
\begin{gathered}
\sigma_{r r}=2 \frac{\partial u}{\partial r}+\left(\beta^{2}-2\right) e-\beta^{2}\left(1+\nu \frac{\partial}{\partial t}\right) \theta \\
\sigma_{\phi \phi}=2 \frac{u}{r}+\left(\beta^{2}-2\right) e-\beta^{2}\left(1+\nu \frac{\partial}{\partial t}\right) \theta \\
\sigma_{z z}=2 \frac{\partial w}{\partial z}+\left(\beta^{2}-2\right) e-\beta^{2}\left(1+\nu \frac{\partial}{\partial t}\right) \theta \\
\sigma_{r z}=\frac{\partial u}{\partial z}+\frac{\partial w}{\partial r}, \\
\beta^{2} \frac{\partial^{2} u}{\partial t^{2}}=\nabla^{2} u-\frac{1}{r^{2}} u+\left(\beta^{2}-1\right) \frac{\partial e}{\partial r}-\beta^{2}\left(1+\nu \frac{\partial}{\partial t}\right) \frac{\partial \theta}{\partial r}, \\
\beta^{2} \frac{\partial^{2} w}{\partial t^{2}}=\nabla^{2} w+\left(\beta^{2}-1\right) \frac{\partial e}{\partial z}-\beta^{2}\left(1+\nu \frac{\partial}{\partial t}\right) \frac{\partial \theta}{\partial z}, \\
\nabla^{2} \theta=\left(\frac{\partial}{\partial t}+\tau_{0} \frac{\partial^{2}}{\partial t^{2}}\right) \theta+\varepsilon\left(\frac{\partial}{\partial t}+n_{0} \tau_{0} \frac{\partial^{2}}{\partial t^{2}}\right) e
\end{gathered}
$$

where

$$
\beta^{2}=\frac{\lambda+2 \mu}{\mu}, \quad \varepsilon=\frac{T_{0} \gamma^{2}}{\rho C_{E}(\lambda+2 \mu)} .
$$

In axisymmetric problems, we use the Helmholtz decomposition as [15]

$$
\mathbf{u}=\operatorname{grad} \varphi+\operatorname{curl}\left(0,-\frac{\partial \psi}{\partial r}, 0\right)
$$

The functions $\varphi$ and $\psi$, respectively, represent the dilatational and rotational parts of $\mathbf{u}$. From (2.16), it follows that (2.12)-(2.14) are identically satisfied if $\varphi$ and $\psi$ satisfy

$$
\begin{gathered}
\left(\nabla^{2}-\frac{\partial^{2}}{\partial t^{2}}\right) \varphi-\left(1+\nu \frac{\partial}{\partial t}\right) \theta=0, \\
\left(\nabla^{2}-\beta^{2} \frac{\partial^{2}}{\partial t^{2}}\right) \psi=0, \\
\left(\nabla^{2}-\frac{\partial}{\partial t}-\tau_{0} \frac{\partial^{2}}{\partial t^{2}}\right) \theta-\varepsilon\left(\frac{\partial}{\partial t}+n_{0} \tau_{0} \frac{\partial^{2}}{\partial t^{2}}\right) \nabla^{2} \varphi=0 .
\end{gathered}
$$

Equation (2.18) for the function $\psi$ is the wave equation with wave velocity $v_{s}=1 / \beta$. This is clearly a shear (transverse) wave and has no effect on the temperature. Roughly speaking, if $\theta$ is considered to be a known function, then (2.17) is the equation of an elastic dilatational compressional wave moving with the velocity $v_{e}=1$. Also, considering $\varphi$ to be a known function, (2.19) is the wave of the temperature $\theta$. This signifies a longitudinal thermal wave moving with the velocity $v_{t}=1 / \sqrt{\tau_{0}}$. The actual situation is, of course, more complicated due to the coupling between the temperature and the dilatation. 
We will assume that the initial state is quiescent, that is, that all the initial conditions of the problem are homogeneous. The boundary conditions of the problem are taken as

$$
\theta(r, \pm h, t)=\theta_{0}(r) H(t), \quad \sigma_{z z}(r, \pm h, t)=\sigma_{r z}(r, \pm h, t)=0
$$

where $H(t)$ is the Heaviside unit step function.

\section{Solution in the transform domain}

We introduce the Laplace transform defined by the relation

$$
\bar{f}(s)=\mathfrak{E}[f(t)]=\int_{0}^{\infty} e^{-s t} f(t) d t
$$

and the Hankel transform defined by the relation

$$
f^{*}(\alpha)=\hbar[f(r)]=\int_{0}^{\infty} f(r) r J_{0}(\alpha r) d r
$$

where $J_{0}$ is the Bessel function of the first kind of order zero. Using the relation [5]

$$
\hbar\left[\frac{\partial^{2} f}{\partial r^{2}}+\frac{1}{r} \frac{\partial f}{\partial r}\right]=-\alpha^{2} f^{*}(\alpha)
$$

and taking the Laplace and Hankel transforms of both sides of (2.17)-(2.19), we obtain

$$
\begin{gathered}
\left(D^{2}-\alpha^{2}-s^{2}\right) \bar{\varphi}^{*}-(1+\nu s) \bar{\theta}^{*}=0, \\
\left(D^{2}-\alpha^{2}-\beta^{2} s^{2}\right) \bar{\psi}^{*}=0, \\
\left(D^{2}-\alpha^{2}-s-\tau_{0} s^{2}\right) \bar{\theta}^{*}-\varepsilon s\left(1+n_{0} \tau_{0} s\right)\left(D^{2}-\alpha^{2}\right) \bar{\varphi}^{*}=0,
\end{gathered}
$$

where the operator $D$ denotes partial differentiation with respect to $z$. Eliminating $\bar{\theta}^{*}$ between (3.4) and (3.6), we obtain the following fourth-order equation satisfied by $\bar{\varphi}^{*}$ :

$$
\left(D^{2}-k_{1}^{2}\right)\left(D^{2}-k_{2}^{2}\right) \bar{\varphi}^{*}=0,
$$

where $\pm k_{1}$ and $\pm k_{2}$ are the roots of the characteristic equation

$$
k^{4}-\left[p(s)+2 \alpha^{2}\right] k^{2}+\alpha^{2}\left(p(s)+\alpha^{2}\right)+s^{3}\left(1+\tau_{0} s\right)=0,
$$

where

$$
p(s)=s^{2}+s\left(1+\tau_{0} s\right)+\varepsilon s\left(1+n_{0} \tau_{0} s\right)(1+\nu s) .
$$


1020 Discontinuities in an axisymmetric thermoelastic problem

It follows from the symmetry of the problem that the solutions of (3.5)-(3.7) with respect to $z$ have the form

$$
\begin{gathered}
\bar{\varphi}^{*}=\sum_{n=1}^{2} A_{n} \cosh k_{n} z, \\
\bar{\psi}^{*}=C \sinh q z, \\
\bar{\theta}^{*}=\sum_{n=1}^{2} \frac{k_{n}^{2}-\alpha^{2}-s^{2}}{1+\nu s} A_{n} \cosh k_{n} z,
\end{gathered}
$$

where $q^{2}=\alpha^{2}+\beta^{2} s^{2}$. The parameters $A_{1}, A_{2}$, and $C$ depend on $s$ and $\alpha$. Using (2.10), (2.11), (2.14), and (3.3), the stress components $\bar{\sigma}_{z z}^{*}$ and $\bar{\sigma}_{r z}^{*}$ take the form

$$
\begin{gathered}
\bar{\sigma}_{z z}^{*}=\left(\beta^{2} s^{2}+2 \alpha^{2}\right) \bar{\varphi}^{*}+2 \alpha^{2} \frac{\partial \bar{\psi}^{*}}{\partial z}, \\
\bar{\sigma}_{r z}^{*}=\hbar\left[\frac{\partial}{\partial r}\left[2 D \bar{\varphi}+\left(2 D^{2}-\beta^{2} s^{2}\right) \bar{\psi}\right]\right] .
\end{gathered}
$$

The boundary conditions (2.20), together with (3.10c) and (3.11), yield

$$
\begin{aligned}
& \sum_{n=1}^{2}\left(k_{n}^{2}-\alpha^{2}-s^{2}\right) A_{n} \cosh k_{n} h=\frac{1}{s}(1+v s) \theta_{0}^{*}(\alpha), \\
& \left(\beta^{2} s^{2}+2 \alpha^{2}\right) \sum_{n=1}^{2} A_{n} \cosh k_{n} h+2 \alpha^{2} q C \cosh q h=0 \\
& 2 \sum_{n=1}^{2} k_{n} A_{n} \sinh k_{n} h+\left(2 q^{2}-\beta^{2} s^{2}\right) C \sinh q h=0 .
\end{aligned}
$$

Equations (3.12) constitute a system of linear algebraic equations in the unknown parameters $A_{1}, A_{2}$, and $C$. The solution of this system is given by

$$
\begin{aligned}
A_{n} & =(-1)^{n} \frac{(1+v s)\left(4 \alpha^{2} q k_{3-n} \tanh \left(k_{3-n} h\right)-\left(2 \alpha^{2}+\beta^{2} s^{2}\right)^{2} \tanh q h\right)}{s \Lambda\left(2 \alpha^{2}+\beta^{2} s^{2}\right)^{2} \tanh q h \cosh k_{n} h} \theta_{0}^{*}(\alpha), \\
C & =2 \frac{(1+v s)\left(k_{2} \tanh k_{2} h-k_{1} \tanh k_{1} h\right)}{s \Lambda\left(2 \alpha^{2}+\beta^{2} s^{2}\right) \sinh q h} \theta_{0}^{*}(\alpha), \\
\Lambda & =k_{1}^{2}-k_{2}^{2}+\frac{4 \alpha^{2} q}{\left(2 \alpha^{2}+\beta^{2} s^{2}\right)^{2} \tanh q h} \sum_{n=1}^{2}(-1)^{n-1} k_{n}\left(k_{3-n}^{2}-\alpha^{2}-s^{2}\right) \tanh k_{n} h .
\end{aligned}
$$

\section{Analysis of discontinuities}

In this section, we use an exact method developed by Boley [2] to determine the magnitude of the propagating jump discontinuities in functions fields, wavefronts, and speeds. The advantage of Boley's method is that it extracts time-domain information directly from Laplace transform expressions without actually inverting these expressions. The following theorem [2] is especially useful when the Laplace transform includes exponential functions. 
THEOREM 4.1. Let $f(t)$ be the inverse Laplace transform of a function $\bar{f}(s)$ :

$$
f(t)=\mathfrak{L}^{-1}[\bar{f}(s)]=\frac{1}{2 \pi i} \int_{d-i \infty}^{d+i \infty} \bar{f}(s) e^{g(s, t)} d s .
$$

If for large s there exists

$$
\bar{f}(s)=\frac{K}{s^{m}}\left[1-\mathrm{O}\left(\frac{1}{s}\right)\right], \quad m>0
$$

and if there exists a function $\zeta(t)$ such that for large s,

$$
g(s, t)-s \zeta(t)=\mathrm{O}\left(\frac{1}{s}\right)
$$

then the discontinuity of $f(t)$ is given by

$$
[f(t)]=f(t+0)-f(t-0)= \begin{cases}0 & \text { for } \zeta \neq 0, \\ 0 & \text { if } m>1 \text { and for } \zeta=0 \\ K & \text { if } m=1 \text { and for } \zeta=0 \\ \infty & \text { if } m<1 \text { and for } \zeta=0 .\end{cases}
$$

To use this theorem, we will expand all the relevant quantities in powers of $1 / s$. From (3.8), $k_{1}$ and $k_{2}$ are given by

$$
k_{n}^{2}=\alpha^{2}+\frac{1}{2}\left(p(s)+(-1)^{n-1} \sqrt{p(s)^{2}-4 s^{3}\left(1+\tau_{0} s\right)}\right) .
$$

Expanding $k_{n}$ in a Maclaurin series and retaining only the first three terms, we obtain

$$
k_{n}^{2}=s^{2}\left(a_{n 0}+\frac{a_{n 1}}{s}+\frac{a_{n 2}}{s^{2}}+\cdots\right), \quad n=1,2,
$$

where

$$
\begin{gathered}
a_{10}=\frac{1}{2}\left[1+\tau_{0}\left(1+n_{0} \varepsilon\right)+\nu \varepsilon+a\right], \\
a_{11}=\frac{1+\varepsilon}{2 a}\left[a+1+\tau_{0}\left(1+n_{0} \varepsilon\right)+\nu \varepsilon\right]-\frac{1}{a}, \\
a_{12}=\alpha^{2}+\frac{\varepsilon}{a^{3}}\left[1+\left(1-n_{0}\right)(1+\varepsilon)(\nu-\tau)\right], \\
a_{20}=a_{10}-a, \\
a_{21}=1+\varepsilon-a_{11}, \\
a_{22}=\alpha^{2}-\frac{\varepsilon}{a^{3}}\left[1+\left(1-n_{0}\right)(1+\varepsilon)(\nu-\tau)\right], \\
a=\left[\left(1+\left(1+n_{0} \varepsilon\right) \tau_{0}+\varepsilon \nu\right)^{2}-4 \tau_{0}\right]^{1 / 2} .
\end{gathered}
$$


1022 Discontinuities in an axisymmetric thermoelastic problem

Using the Maclaurin expansion a second time, we obtain

$$
k_{n}=s\left(b_{n 0}+\frac{b_{n 1}}{s}+\frac{b_{n 2}}{s^{2}}+\cdots\right), \quad n=1,2,
$$

where

$$
b_{n 0}=a_{n 0}^{1 / 2}, \quad b_{n 1}=\frac{1}{2} \frac{a_{n 1}}{a_{n 0}^{1 / 2}}, \quad b_{n 2}=\frac{4 a_{n 2} a_{n 0}-a_{n 1}^{2}}{8 a_{n 0}^{3 / 2}} .
$$

Using similar expansion techniques, we note that for large $s$, we have

$$
\begin{gathered}
\tanh k_{n}=\tanh q h=1+\mathrm{O}\left(\frac{1}{s}\right), \\
\cosh k_{n} h=\frac{1}{2} e^{k_{n} h}+\mathrm{O}\left(\frac{1}{s}\right), \quad \sinh q h=\frac{1}{2} e^{q h}+\mathrm{O}\left(\frac{1}{s}\right), \\
k_{1}^{2}-k_{2}^{2}=a s^{2}+\mathrm{O}(s), \quad q=\beta s+\mathrm{O}\left(\frac{1}{s}\right), \\
A_{n}=2(-1)^{n+1} \frac{(1+v s) \theta_{0}^{*}(\alpha)}{a s^{3}} e^{-k_{n} h}+\mathrm{O}\left(\frac{1}{s^{4}}\right), \\
C=4 \frac{(1+v s)\left(b_{20}-b_{10}\right) \theta_{0}^{*}(\alpha)}{a \beta^{2} s^{4}} e^{-\beta s h}+\mathrm{O}\left(\frac{1}{s^{5}}\right) .
\end{gathered}
$$

Collecting the previous results, (3.10c) for large $s$ takes the form (for the three theories)

$$
\begin{aligned}
\bar{\theta}(r, z, s)= & \frac{1}{s} \frac{a_{10}-1}{a} \int_{0}^{\infty} \alpha J_{0}(\alpha r) \theta_{0}^{*}(\alpha) e^{k_{1}(z-h)} d \alpha \\
& +\frac{1}{s} \frac{a_{10}-1}{a} \int_{0}^{\infty} \alpha J_{0}(\alpha r) \theta_{0}^{*}(\alpha) e^{-k_{1}(z+h)} d \alpha \\
& -\frac{1}{s} \frac{a_{20}-1}{a} \int_{0}^{\infty} \alpha J_{0}(\alpha r) \theta_{0}^{*}(\alpha) e^{k_{2}(z-h)} d \alpha \\
& -\frac{1}{s} \frac{a_{20}-1}{a} \int_{0}^{\infty} \alpha J_{0}(\alpha r) \theta_{0}^{*}(\alpha) e^{-k_{2}(z+h)} d \alpha+\mathrm{O}\left(\frac{1}{s^{2}}\right)
\end{aligned}
$$

The inverse Laplace of the first term of the last equation is given by

$$
I_{1}=\frac{1}{2 \pi i} \int_{d-i \infty}^{d+i \infty} \frac{1}{s} \frac{a_{10}-1}{a} \int_{0}^{\infty} \alpha J_{0}(\alpha r) \theta_{0}^{*}(\alpha) e^{k_{1}(z-h)+s t} d \alpha d s
$$

Choosing $g(s, t)=k_{1}(z-h)+s t$ and $\zeta(t)=b_{10}(z-h)+t$, and using (4.8) for $k_{1}$, we finally obtain

$$
I_{1}=\frac{1}{2 \pi i} \int_{d-i \infty}^{d+i \infty} \frac{K}{s}\left[1+\mathrm{O}\left(\frac{1}{s}\right)\right] e^{g(s, t)} d s
$$


Table 4.1. Propagating discontinuities in $\theta$, where $I_{0}=(1 / a) \int_{0}^{\infty} \alpha J_{0}(\alpha r) \theta_{0}^{*}(\alpha) d \alpha$.

\begin{tabular}{|c|c|c|c|}
\hline Theories & & & CT \\
\hline Wavefronts & Elastic & Thermal & Elastic \\
\hline$[\theta]$ & $\left(a_{10}-1\right) I_{0} e^{-t b_{11} / b_{10}}$ & $\left(1-a_{20}\right) I_{0} e^{-t b_{21} / b_{20}}$ & 0 \\
\hline
\end{tabular}

with $g(s, t)-s \zeta(t)=\mathrm{O}(1 / s)$ and

$$
[\theta]=K=\frac{a_{10}-1}{a} e^{b_{11}(z-h)} \int_{0}^{\infty} \alpha J_{0}(\alpha r) \theta_{0}^{*}(\alpha) d \alpha .
$$

This is in the form of (4.4) with $m=1$. This means that the function has a finite discontinuity of size $K$ when $\zeta(t)=0$, that is, when $t+b_{10}(z-h)=0$. This is the equation of a wave moving from the upper surface $(z=h)$ with a velocity equal to $1 / b_{10}$. This wave is mainly elastic in nature, and its velocity is obtained from (4.6),

$$
v_{e}=\sqrt{\frac{2}{1+\tau_{0}\left(1+n_{0} \varepsilon\right)+\nu \varepsilon+a}},
$$

and arrives at the middle plane in time equal to

$$
h \sqrt{\frac{\left[1+\tau_{0}\left(1+n_{0} \varepsilon\right)+\nu \varepsilon+a\right]}{2}} .
$$

Similarly, the second term in the right-hand side of the last expression of $\bar{\theta}$ represents a wave moving from the lower surface of the plate $(z=-h)$ with the same speed. The third term represents a wave moving with a velocity equal to $1 / b_{20}$. This velocity approaches $1 / \sqrt{\tau_{0}}$ as $\varepsilon \rightarrow 0$. It is clear that under the classical theory $\left(\tau_{0}=v=0\right)$, this wave propagates with infinite velocity. Thus, this wave is mainly the thermal wave mentioned above. The exact value of the velocity is

$$
v_{t}=\sqrt{\frac{2}{1+\tau_{0}\left(1+n_{0} \varepsilon\right)+\nu \varepsilon-a}} .
$$

This wave arrives at the middle plane in time equal to

$$
h \sqrt{\frac{\left[1+\tau_{0}\left(1+n_{0} \varepsilon\right)+\nu \varepsilon-a\right]}{2}} .
$$

Under LS/GL theories, it is clear that $\theta$ experiences finite jump across both elastic and thermal wavefronts, which decays exponentially over time (see Table 4.1).

Furthermore, by applying the same procedure to the first displacement component $u$ for LS/CT theories, it is found that $u$ being $\mathrm{O}\left(1 / s^{3}\right)(m>1)$ is a continuous function together with its first derivatives (see Table 4.2). The jump in the value of $\partial u / \partial t$ is found by applying Boley's method and using the well-known relation $\mathfrak{E}[\partial u / \partial t]=s \mathfrak{E}[u]$. For GL theory, it is found that $\partial u / \partial t$ has a finite discontinuity of size $K$ when $\zeta(t)=0$, that is, 
1024 Discontinuities in an axisymmetric thermoelastic problem

Table 4.2. Propagating discontinuities in $u$ and its first derivatives, where $\Gamma=-2\left(b_{20}-b_{10}\right) / \beta, I_{1}=1$ / $a \int_{0}^{\infty} \alpha^{2} J_{1}(\alpha r) \theta_{0}^{*}(\alpha) d \alpha$.

\begin{tabular}{lccccccc}
\hline Theories & \multicolumn{3}{c}{ LS } & \multicolumn{2}{c}{ GL } & \multicolumn{2}{c}{ CT } \\
\hline Wavefronts & Elastic & Thermal & Shear & Elastic & Thermal & Shear & Elastic \\
\hline$[u]$ & 0 & 0 & 0 & 0 & 0 & 0 & 0 \\
{$[\partial u / \partial t]$} & 0 & 0 & 0 & $-\nu I_{1} e^{-t b_{11} / b_{10}}$ & $\nu I_{1} e^{-t b_{21} / b_{20}}$ & $\nu I_{1} \Gamma$ & 0 \\
{$[\partial u / \partial z]$} & 0 & 0 & 0 & $-\nu b_{10} I_{1} e^{-t b_{11} / b_{10}}$ & $\nu b_{20} I_{1} e^{-t b_{21} / b_{20}}$ & $\nu I_{1} \beta \Gamma$ & 0 \\
{$[\partial u / \partial r]$} & 0 & 0 & 0 & 0 & 0 & 0 & 0 \\
\hline
\end{tabular}

Table 4.3. Propagating discontinuities in $w$ and its first derivatives.

\begin{tabular}{lccccc}
\hline Theories & \multicolumn{2}{c}{ LS } & \multicolumn{2}{c}{ GL } & CT \\
\hline Wavefronts & Elastic & Thermal & Elastic & Thermal & Elastic \\
\hline$[w]$ & 0 & 0 & $\nu b_{10} I_{0} e^{-t b_{11} / b_{10}}$ & $-v b_{20} I_{0} e^{-t b_{21} / b_{20}}$ & 0 \\
{$[\partial w / \partial t]$} & $b_{10} I_{0} e^{-t b_{11} / b_{10}}$ & $-b_{20} I_{0} e^{-t b_{21} / b_{20}}$ & $\infty$ & $\infty$ & $I_{0} e^{-\varepsilon t}$ \\
{$[\partial w / \partial z]$} & $a_{10} I_{0} e^{-t b_{11} / b_{10}}$ & $-a_{20} I_{0} e^{-t b_{21} / b_{20}}$ & $\infty$ & $\infty$ & $I_{0} e^{-\varepsilon t}$ \\
{$[\partial w / \partial r]$} & 0 & 0 & $-v b_{10} I_{1} e^{-t b_{11} / b_{10}}$ & $\nu b_{20} I_{1} e^{-t b_{21} / b_{20}}$ & 0 \\
\hline
\end{tabular}

when $\beta(z-h)+t=0$. This is the equation of a wave moving from the upper surface $(z=h)$ with a velocity equal to $v_{s}=1 / \beta$. This is clearly a shear (transverse) wave mentioned above. We note that the magnitude of the jump across this wavefront does not decay exponentially over time. Finally, under GL theory, $u$ and $\partial u / \partial r$ being $O\left(1 / s^{2}\right)$ are continuous functions, but $\partial u / \partial t$ and $\partial u / \partial z$ experience finite discontinuities at the three wavefronts.

For LS/CT theories, the second displacement component $w$ and $\partial w / \partial r$ being $\mathrm{O}\left(1 / s^{2}\right)$ is a continuous function, while $\partial w / \partial t$ and $\partial w / \partial z$ are discontinuous at the elastic and thermal wavefronts (see Table 4.3). Under GL theory, $w$ and its first derivatives are discontinuous across both elastic and thermal wavefronts. All the considered functions in Table 4.3 are continuous at the shear wavefront.

Applying the same procedure to the stress components $\sigma_{r r}, \sigma_{\phi \phi}$, and $\sigma_{z z}$, it is found that these functions suffer a finite discontinuity under LS/CT theories and an infinite discontinuity under GL theory across both elastic and thermal wavefronts. For the generalized theories, $\sigma_{r r}$ and $\sigma_{\phi \phi}$ are continuous, while $\sigma_{z z}$ is discontinuous across the shear wavefront.

\section{Inversion of the double transforms}

We will now outline the numerical method used to find the solution in the physical domain. We make use first of the inversion formula of the Hankel transform [5], namely,

$$
f(r)=\hbar^{-1}\left[f^{*}(\alpha)\right]=\int_{0}^{\infty} f^{*}(\alpha) \alpha J_{0}(\alpha r) d \alpha
$$


Applying this formula to (3.10)-(3.11), we obtain the Laplace transforms

$$
\begin{aligned}
\bar{\theta}(r, z, s) & =\frac{1}{1+\nu s} \int_{0}^{\infty} \alpha J_{0}(\alpha r) \sum_{n=1}^{2}\left(k_{n}^{2}-\alpha^{2}-s^{2}\right) A_{n} \cosh k_{n} z d \alpha, \\
\bar{u}(r, z, s) & =-\int_{0}^{\infty} \alpha^{2} J_{1}(\alpha r) \sum_{n=1}^{2}\left[A_{n} \cosh k_{n} z+C q \cosh q z\right] d \alpha, \\
\bar{w}(r, z, s) & =\int_{0}^{\infty} \alpha J_{0}(\alpha r) \sum_{n=1}^{2}\left[k_{n} A_{n} \sinh k_{n} z+C \alpha^{2} \sinh q z\right] d \alpha, \\
\bar{F} & =\int_{0}^{\infty} \alpha J_{0}(\alpha r) \sum_{n=1}^{2}\left(\beta^{2} s^{2}+2 \alpha^{2}-2 k_{n}^{2}\right) A_{n} \cosh k_{n} z d \alpha, \\
\bar{\sigma}_{r r}(r, z, s) & =\bar{F}+2 \int_{0}^{\infty} \alpha^{3}\left(\frac{1}{\alpha r} J_{1}(\alpha r)-J_{0}(\alpha r)\right) \sum_{n=1}^{2}\left[A_{n} \cosh k_{n}+C q \cosh q z\right] d \alpha, \\
\bar{\sigma}_{\phi \phi}(r, z, s) & =\bar{F}-\frac{2}{r} \int_{0}^{\infty} \alpha^{2} J_{1}(\alpha r) \sum_{n=1}^{2}\left[A_{n} \cosh k_{n}+C q \cosh q z\right] d \alpha, \\
\bar{\sigma}_{z z}(r, z, s) & =\int_{0}^{\infty} \alpha J_{0}(\alpha r)\left(\beta^{2} s^{2}+2 \alpha^{2}\right) \sum_{n=1}^{2}\left[A_{n} \cosh k_{n}+2 \alpha^{2} C \cosh q z\right] d \alpha .
\end{aligned}
$$

In order to invert the Laplace transforms in (5.2)-(5.8), we adopt a numerical inversion method based on a Fourier series expansion [9]. In this method, inversion $f(t)$ of Laplace transform $\bar{f}(s)$ is approximately by the relation

$$
f(t)=\frac{e^{\xi t}}{4 T_{m}}\left[\frac{1}{2} \bar{f}(\xi)+\Re e\left(\sum_{k=1}^{N} e^{i k \pi t / 4 T_{m}} \bar{f}\left(\xi+i \frac{k \pi}{4 T_{m}}\right)\right)\right],
$$

where $N$ is a sufficiently large integer representing the number of terms in the truncated infinite Fourier series, and must be chosen such that

$$
e^{\xi t} \mathfrak{R} e\left(e^{i N \pi t / 4 T_{m}} \bar{f}\left(\xi+i \frac{N \pi}{4 T_{m}}\right)\right) \leq \varepsilon_{0},
$$

where $\varepsilon_{0}$ is a preselected small positive number that corresponds to the degree of accuracy required. The parameter $\xi$ is a positive free parameter that must be greater than the real parts of all singularities of $\bar{f}(s)$. The optimal choice of $\xi$ was obtained according to the criteria described in [9]. $T_{m}$ is the maximum time simulated.

The numerical technique outlined above was used to invert the Laplace transforms in (5.2)-(5.8). The Romberg numerical integration technique [17] with variable step size was used to evaluate the integrals involved.

In order to find temperature distribution $\theta$, we use an expression similar to (5.9) with $\theta$ and $\bar{\theta}$ replacing $f$ and $\bar{f}$, respectively. To illustrate the above results graphically, the axisymmetric function $\theta_{0}(r)$ that is the value of the temperature on the upper and lower surfaces of the plate was chosen to be zero except for the inside of the circular region $r \leq a$ 
where it has a fixed constant value of $c_{0}$, that is,

$$
\theta_{0}(r)=c_{0} H(a-r)
$$

Taking the Hankel transform, we obtain

$$
\theta_{0}^{*}(\alpha)=c_{0} \int_{0}^{\infty} H(a-r) r J_{0}(\alpha r) d r=c_{0} \int_{0}^{a} r J_{0}(\alpha r) d r=\frac{a c_{0}}{\alpha} J_{1}(\alpha a)
$$

The copper material was chosen for purposes of numerical evaluations. The constants of the problem were taken as

$$
\beta^{2}=3.5, \quad \varepsilon=0.0168, \quad \tau_{0}=0.02, \quad v=0.03, \quad a=1, \quad h=1, \quad c_{0}=1 .
$$

Thus, the velocities $v_{t}, v_{e}$, and $v_{s}$ discussed above have the values $v_{t}=7.072, v_{e}=0.999$, and $v_{s}=0.286$. The first is faster than the second and corresponds to the second sound and results from the temperature forcing term in the displacement equations. The thermal wave arrives first in the middle plane after a 0.141 unit of time. This wave is reflected three times with attenuation before the arrival of the elastic wave in 1 unit of time. The computations were carried out for three values of time, namely, $t=0.1,0.2$, and 1.1 , respectively. These values correspond to the middle plane before and after the arrival of the first wave and after the arrival of the second wave, respectively. The temperature $\theta$, the radial displacement component $u$, and the axial stress component $\sigma_{z z}$ are shown in Figures 5.1, 5.2, and 5.3, respectively, and evaluated at the middle of the plane $(z=0)$. Since the displacement component $w$ is an odd function of $z$, its value on the middle plane is always zero (see (5.4)), and it is not represented graphically here. The graphs of the stress components $\sigma_{r r}$ and $\sigma_{\phi \phi}$ are found to be very similar to that of $\sigma_{z z}$ and are omitted here.

We note that the graphs for radial displacement and axial stress distributions do not demonstrate the theoretical predictions of discontinuities. In fact, from Boley's theorem, the function experiences a discontinuity when $\zeta(t)=t+b_{n 0}(z-h)=0$. However, because $\zeta(t) \neq 0$ at $z=0$ for the three considered values of time, discontinuities do not occur. For example, at $t=0.1$, the wavefront (moving at a finite speed) has not reached the middle plane yet under both generalized theories. The solution was found to be identically zero for this value of time at the middle plane for all functions considered. Moreover, due to the dissipative nature of the temperature equation, the magnitude of discontinuity decays exponentially over time (see the tables).

\section{Concluding remarks}

Based on the analysis of discontinuities presented here and numerical results, we state the following conclusions.

(i) It was found from Figures 5.1, 5.2, and 5.3, that for large values of time, the results obtained by using either the classical or the generalized theories are quite similar. The case is quite different when we consider small values of time. Since the classical theory predicts 


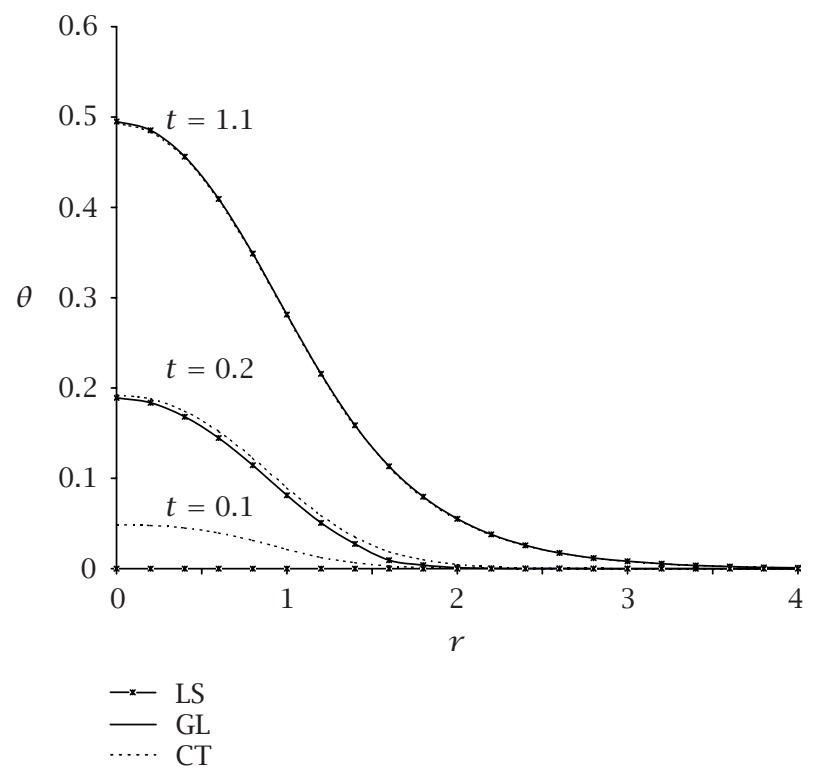

Figure 5.1. Temperature distribution in the middle plane.

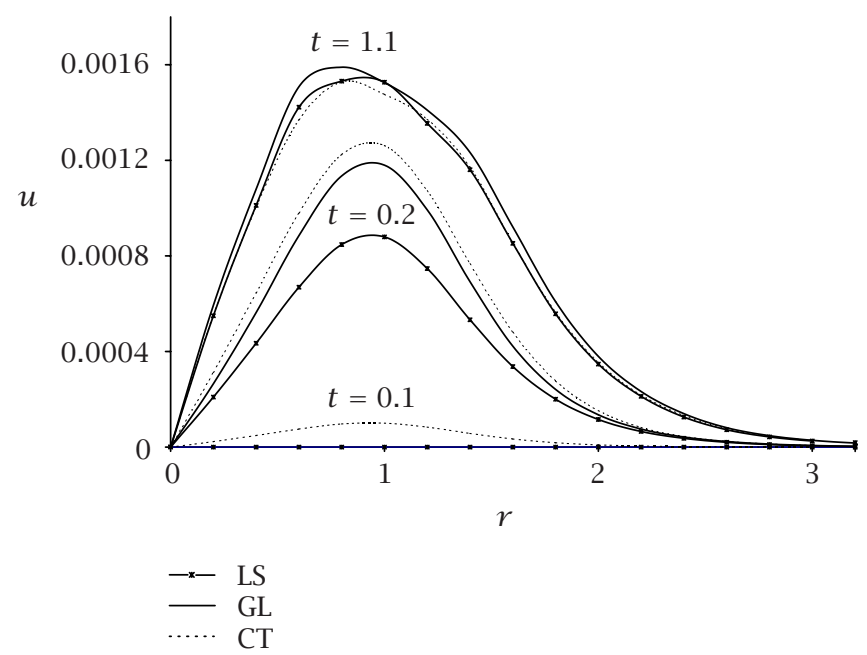

Figure 5.2. Radial displacement distribution in the middle plane.

infinite speeds of wave propagation, the effect of heating at the boundary is transmitted instantaneously to all parts of the medium, so the solution is not identically zero for any value of time (though it may be very small). For the generalized theory, however, the waves take a finite time to be transmitted. This is quite clear in the curve drawn at $t=0.1$ on the radial axis of each figure. 
1028 Discontinuities in an axisymmetric thermoelastic problem

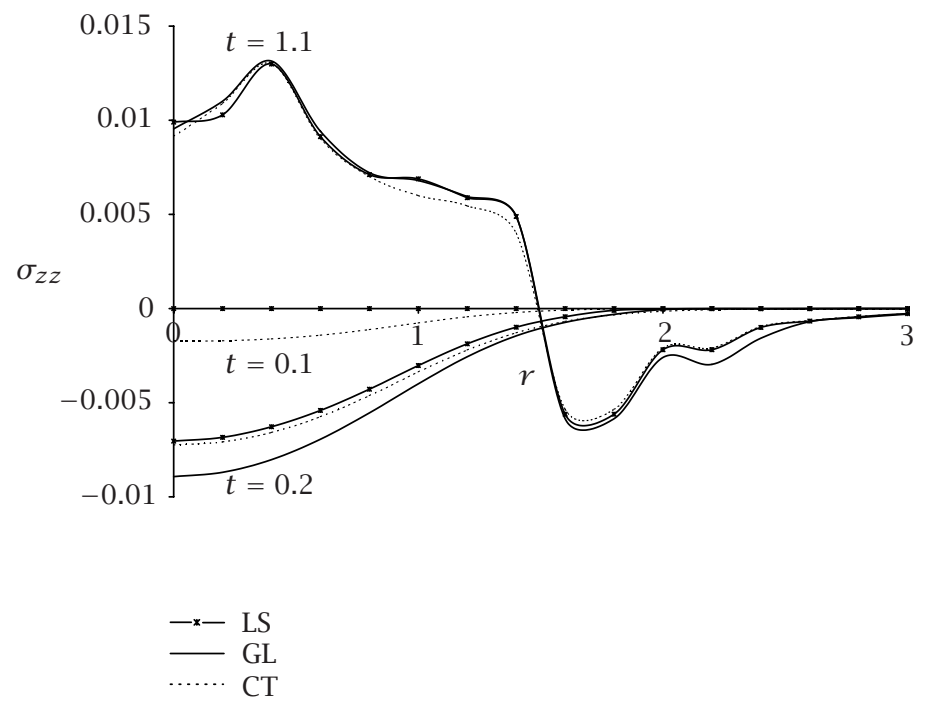

Figure 5.3. Axial stress distribution in the middle plane.

Table 6.1. Propagating discontinuities in stress fields, where $\delta_{1}=\beta^{2}-2 a_{10}, \delta_{2}=-\left(\beta^{2}-2 a_{20}\right), I_{2}=$ $1 / a \int_{0}^{\infty} \alpha^{3} J_{0}(\alpha r) \theta_{0}^{*}(\alpha) d \alpha$.

\begin{tabular}{|c|c|c|c|c|c|c|c|}
\hline Theories & & LS & & & GL & & $\mathrm{CT}$ \\
\hline Wavefronts & Elastic & Thermal & Shear & Elastic & Thermal & Shear & Elastic \\
\hline$\left[\sigma_{r r}\right]$ & $\delta_{1} I_{0} e^{-t b_{11} / b_{10}}$ & $\delta_{2} I_{0} e^{-t b_{21} / b_{20}}$ & 0 & $\infty$ & $\infty$ & 0 & $\lambda / \mu I_{0} e^{-\varepsilon t}$ \\
\hline$\left[\sigma_{\phi \phi}\right]$ & $\delta_{1} I_{0} e^{-t b_{11} / b_{10}}$ & $\delta_{2} I_{0} e^{-t b_{21} / b_{20}}$ & 0 & $\infty$ & $\infty$ & 0 & $\lambda / \mu I_{0} e^{-\varepsilon t}$ \\
\hline$\left[\sigma_{z z}\right]$ & $\beta^{2} I_{0} e^{-t b_{11} / b_{10}}$ & $-\beta^{2} I_{0} e^{-t b_{21} / b_{20}}$ & $-2 I_{2} \beta^{2} \Gamma$ & $\infty$ & $\infty$ & $\infty$ & $\beta^{2} I_{0} e^{-\varepsilon t}$ \\
\hline
\end{tabular}

(ii) From Tables 4.2 and 4.3, the displacement component $u$ is continuous under the three theories of thermoelasticity [14], while $w$ is continuous under LS/CT theories and discontinuous under GL theory [19]. The discontinuity of $w$ under GL theory violates the requirement of continuity of displacements, and implies that one portion of matter penetrates into another [3]. This prediction of GL theory is physically absurd.

(iii) Table 6.1 shows that the magnitudes of discontinuities of the stresses functions are finite under LS theory and infinite under GL theory across both elastic and thermal wavefronts. The same situation arises in the context of LS theory in $[12,14]$ and in the context of GL theory in [12]. This prediction of GL theory is also not physically realistic and supports the a priori Furukawa et al.'s assertion $[6,7]$.

\section{Acknowledgment}

The author would like to thank the reviewers for their critical review and valuable comments which improved the paper thoroughly. 


\section{References}

[1] M. A. Biot, Thermoelasticity and irreversible thermodynamics, J. Appl. Phys. 27 (1956), 240 253.

[2] B. A. Boley, Discontinuities in integral-transform solutions, Quart. Appl. Math. 19 (1962), 273284.

[3] D. S. Chandrasekharaiah and L. Debnath, Continuum Mechanics, Academic Press, Massachusetts, 1994.

[4] D. S. Chandrasekharaiah and K. S. Srinath, Thermoelastic waves without energy dissipation in an unbounded body with a spherical cavity, Int. J. Math. Math. Sci. 23 (2000), no. 8, 555-562.

[5] R. V. Churchill, Operational Mathematics, 3rd ed., McGraw-Hill Book Company, New York, 1972.

[6] T. Furukawa, N. Noda, and F. Ashida, Generalized thermoelasticity for an infinite body with a circular cylindrical hole, JSME Int. J. 33 (1990), 26-32.

[7] _ Generalized thermoelasticity for an infinite solid cylinder, JSME Int. J. 34 (1991), 281286.

[8] A. E. Green and K. A. Lindsay, Thermoelasticity, J. Elasticity 2 (1972), 1-7.

[9] G. Honig and U. Hirdes, A method for the numerical inversion of Laplace transforms, J. Comput. Appl. Math. 10 (1984), no. 1, 113-132.

[10] J. Ignaczak, Decomposition theorem for thermoelasticity with finite wave speeds, J. Thermal Stresses 1 (1978), 41-52.

[11] A strong discontinuity wave in thermoelasticity with relaxation times, J. Thermal Stresses 8 (1985), no. 1, 25-40.

[12] P. M. Jordan and P. Puri, Thermal stresses in a spherical shell under three thermoelastic models, J. Thermal Stresses 24 (2001), 47-70.

[13] H. Lord and Y. Shulman, A generalized dynamical theory of thermoelasticity, J. Mech. Phys. 15 (1967), 299-309.

[14] B. Mukhopadhyay, R. Bera, and L. Debnath, On generalized thermoelastic disturbances in an elastic solid with a spherical cavity, J. Appl. Math. Stochastic Anal. 4 (1991), 225-240.

[15] W. Nowacki, Dynamic Problems of Thermoelasticity, Noordhoff International Publishing, 1975.

[16] I. R. Orisamolu, M. N. K. Singh, and M. C. Singh, Propagation of coupled thermomechanical waves in uniaxial inelastic solids, J. Thermal Stresses 25 (2002), 927-949.

[17] W. H. Press, B. P. Flannery, S. A. Teukolsky, and W. T. Vetterling, Numerical Recipes, Cambridge University Press, Cambridge, 1986, the art of scientific computing.

[18] Yu. A. Rossikhin and M. V. Shitikova, The impact of a thermoelastic rod against a rigid heated barrier, J. Engrg. Math. 44 (2002), no. 1, 83-103.

[19] S. K. Roychoudhuri and G. Chatterjee, Spherically symmetric thermoelastic waves in a temperature rate dependent medium with spherical cavity, Comput. Math. Appl. 20 (1990), no. 11, $1-12$.

Moncef Aouadi: Department of Mathematics and Computer Science, Rustaq Faculty of Education, Rustaq 329, P.O. Box 10, Sultanate of Oman

E-mail address: moncef_aouadi@yahoo.fr 


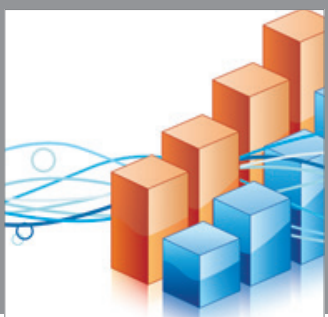

Advances in

Operations Research

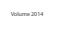

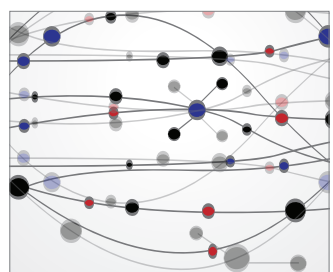

\section{The Scientific} World Journal
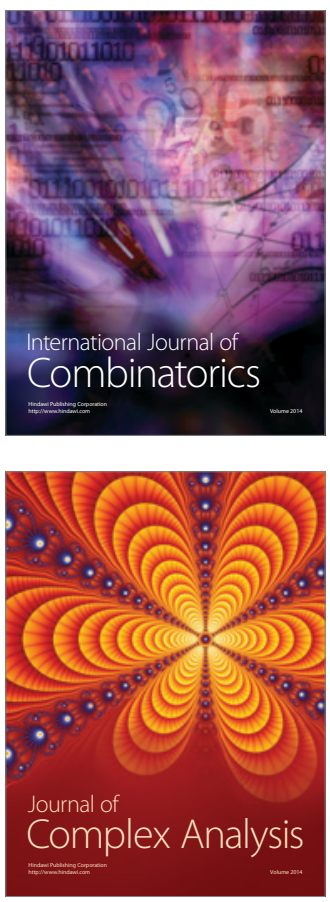

International Journal of

Mathematics and

Mathematical

Sciences
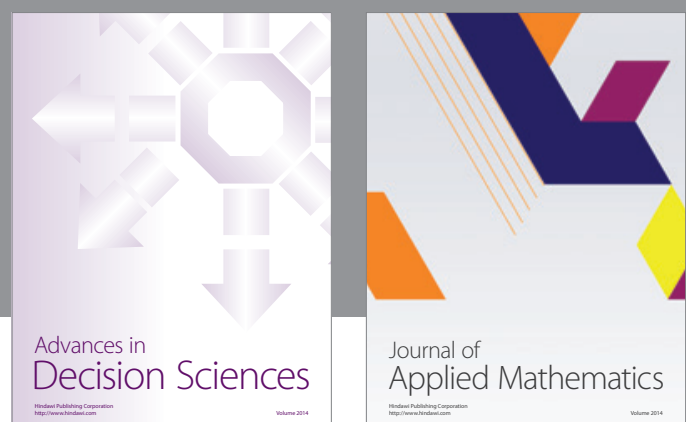

Journal of

Applied Mathematics
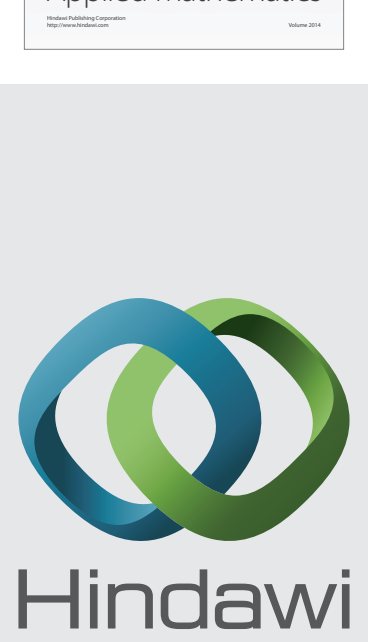

Submit your manuscripts at http://www.hindawi.com
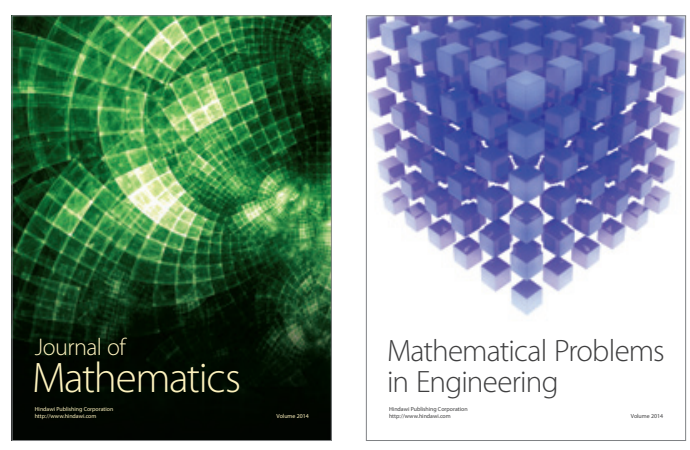

Mathematical Problems in Engineering
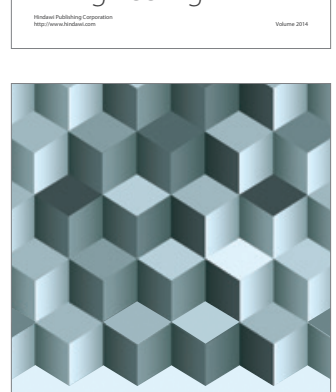

Journal of

Function Spaces
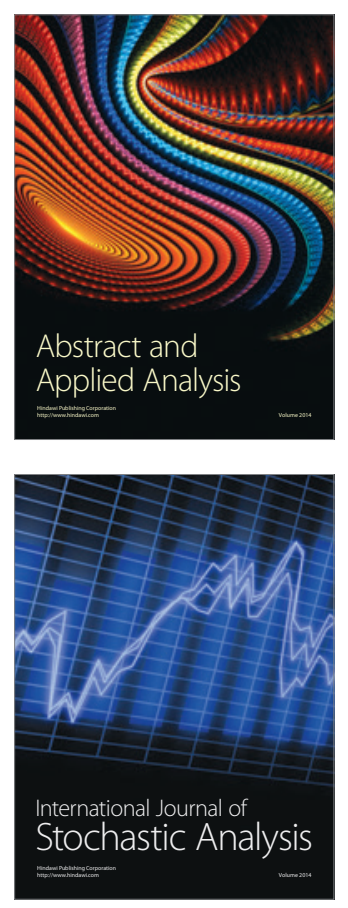

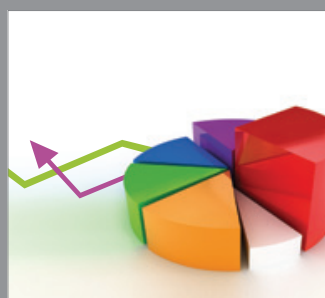

ournal of

Probability and Statistics

Promensencen
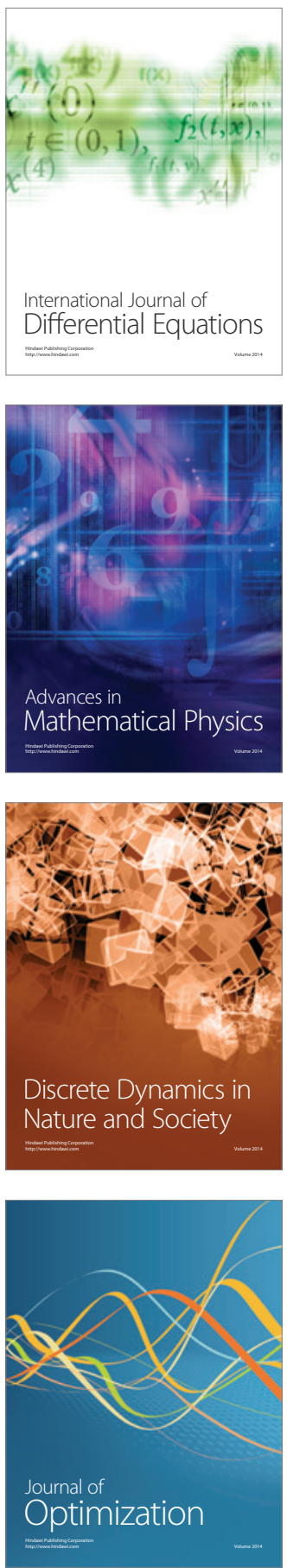